\title{
Erratum to: Is prenatal myo-inositol deficiency a mechanism of CNS injury in galactosemia?
}

\author{
Gerard T. Berry
}

Published online: 11 March 2011

(C) SSIEM and Springer 2011

\section{Erratum to: J Inherit Metab Dis DOI 10.1007/s10545-010-9260-x}

Unfortunately, in the original published article, there are some errors. The following paragraphs should read:

\section{Heading Evidence for myo-inositol deficiency}

Lines "In high concentrations, galactose-1-phosphate may ... to .... Myo-inositol transporter activity" should read: In high concentrations, galactose-1 phosphate may inhibit myo-inositol recycling in developing neurons following agonist-induced signal transduction events, as it may lead to trapping of myo-inositol as inositol monophosphate. Independently, intracellular galactitol accumulation via osmoregulatory perurbations may reduce myo-inositol transporter activity in fetal neurons.

\section{Heading Physiology and biochemistry of myo-inositol}

Lines "Almost all of the PtdIns-4,5- $\mathrm{P}_{2} \ldots$ to .... (Berridge and Irvine 1989)." should read:

Almost all of the PtdIns-4,5- $\mathrm{P}_{2}$ that is involved in membrane signaling events, especially in the CNS, contains arachidonate in the $s n 1$ position and stearate in the $s n 2$ position. Following agonist-induced receptor activation, PtdIns-4,5- $\mathrm{P}_{2}$ is hydrolyzed by a phospholipase $\mathrm{C}$ to myoinositol-1,4,5-trisphosphate (Ins-1,4,5- $\mathrm{P}_{3}$ ) and diacylglycerol. Subsequently, the water soluble second messenger, Ins-1,4,5- $\mathrm{P}_{3}$, binds to an Ins-1,4,5- $\mathrm{P}_{3}$ receptor on an internal membrane to activate a calcium channel resulting in a transient burst in cytosolic calcium activity (Berridge and Irvine 1989).

\section{Heading Physiology and biochemistry of myo-inositol} Lines "Also, PtdIns deficiency may lead to impaired ... to ... Loretscher and Lavery 2002)." should read:

Also, PtdIns deficiency may lead to impaired synthesis of ectoproteins that are anchored to the plasma membrane via PtdIns such as alkaline phosphatase, 5'-nucleotidase, acetylcholinesterase, folate receptor and Thy-1 (Sharom and Lehto 2002; Loretscher and Lavery 2002).

In the legend to Fig. 4, last two lines, it should read:

... sodium/myo-inositol cotransporter1. (not ... sodium/ myo-inositolcotransporter1.)

The online version of the original article can be found at http://dx.doi. org/10.1007/s10545-010-9260-x.

G. T. Berry $(\bowtie)$

Division of Genetics, Children's Hospital Boston,

Harvard Medical School,

Center for Life Sciences Building, Room 14070

3 Blackfan Circle,

Boston, MA 02115, USA

e-mail: Gerard.Berry@childrens.harvard.edu 